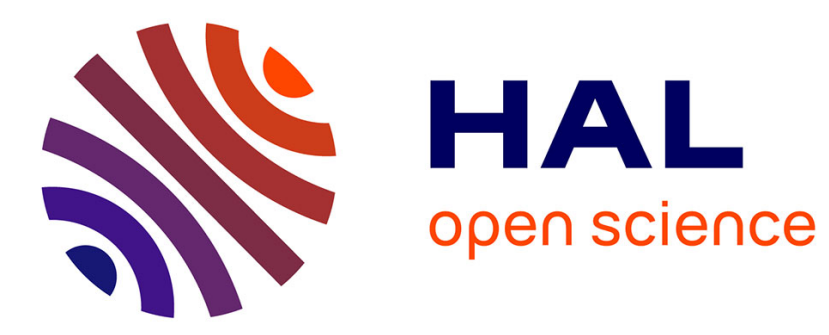

\title{
The G0 experiment at Jefferson Laboratory: The nucleon strangeness form factors \\ C. Furget
}

\section{To cite this version:}

C. Furget. The G0 experiment at Jefferson Laboratory: The nucleon strangeness form factors. 4th International Workshop on Neutrino-Nucleus Interactions in the Few-GeV Region - NuInt'05, Sep 2005, Okayama, Japan. pp.121-126, 10.1016/j.nuclphysbps.2006.08.072 . in2p3-00025620

HAL Id: in2p3-00025620

https://hal.in2p3.fr/in2p3-00025620

Submitted on 14 Feb 2006

HAL is a multi-disciplinary open access archive for the deposit and dissemination of scientific research documents, whether they are published or not. The documents may come from teaching and research institutions in France or abroad, or from public or private research centers.
L'archive ouverte pluridisciplinaire HAL, est destinée au dépôt et à la diffusion de documents scientifiques de niveau recherche, publiés ou non, émanant des établissements d'enseignement et de recherche français ou étrangers, des laboratoires publics ou privés. 


\title{
The $\mathrm{G}^{0}$ experiment at Jefferson Laboratory : The nucleon strangeness form factors
}

\author{
C. Furget ${ }^{\mathrm{a}}$, for the $\mathrm{G}^{0}$ collaboration * \\ ${ }^{a}$ Laboratoire de Physique Subatomique et de Cosmologie, \\ 53 avenue des Martyrs, 38026 Grenoble Cedex, France
}

The $G^{0}$ experiment is dedicated to the determination of the strange quark's contribution to the electric and magnetic nucleon form factors, provided by parity-violating asymmetries of cross-sections measured with longitudinaly polarized electrons in elastic electron-proton scattering and quasi-elastic electron-deuteron scattering. Forward angle measurements, which have been performed in Hall C of Jefferson Laboratory, have provided a linear combination of electric and magnetic vector form factors for momentum transfers in the range 0.1 to $1(\mathrm{GeV} / \mathrm{c})^{2}$. Backward angle measurements, which will be performed starting in 2006, will allow the complete separation of the form factors at $Q^{2}$ of 0.23 and $0.63(\mathrm{GeV} / \mathrm{c})^{2}$.

\section{Introduction}

In Quantum Chromodynamics, the nucleon is viewed as composed of three valence $u$ and $d$ quarks immersed in a sea of gluons and quarkantiquark pairs $(u \bar{u}, d \bar{d}, s \bar{s} \ldots)$. The study of the contribution of strange quarks to the nucleon properties provides useful information on the sea itself. First indications already exist, namely the strange quarks contribution to the proton momentum and the spin from neutrino and electron deep inelastic scattering and to its mass from $\pi$-nucleon scattering. Their contributions range from 0 to $30 \%$ with large theoretical and experimental uncertainties.

The parity-violating (PV) electro-weak interaction in electron-nucleon elastic scattering offers a new opportunity to study the contribution of strange quarks to the electro-weak structure of the nucleon through the nucleon vector form factors and the axial form factor, the latter also being accessible from neutrino elastic scattering [13]. Several dedicated PV asymmetry experiments and apparatus have been developed during the last decade for these studies $[4,5]$. In this contribution we focus on the $G^{0}$ experiment which will provide a complete determination of the contribution of the strange quarks to the electric, mag- netic and axial form factors for several momentum transfers.

In the following, we will present the physics motivation before describing the $G^{0}$ experimental set-up. Then we will show the first results of the $G^{0}$ experiment obtained in the forward angle kinematics which provide a linear combination of the strange contribution to the electric and magnetic nucleon form factors over a large $Q^{2}$ range between 0.12 to $1(\mathrm{GeV} / \mathrm{c})^{2}$.

\section{Elastic scattering and parity violating experiment}

In the electron-nucleon scattering, the electroweak interaction takes place at first order through two scattering amplitudes, one corresponding to the exchange of a virtual photon $\left(\mathcal{M}_{\gamma}\right)$ and one to the exchange of a $Z^{0}\left(\mathcal{M}_{Z}\right)$, which depend on the 4-momentum transferred $Q^{2}$, which is related to the distance scale probed in the nucleon. The scattering amplitudes can be decomposed in terms of the electromagnetic proton form factors $G_{E}^{(\gamma, p)}$ and $G_{M}^{(\gamma, p)}$ and the electric $G_{E}^{(Z, p)}$, magnetic $G_{M}^{(Z, p)}$ and axial $G_{A}^{e p}$ weak form factors. In order to provide information on the spatial distribution of the quarks inside the nucleon, each of the nucleon form factors is ex- 
pressed in terms of its quark contribution as :

$$
\begin{aligned}
G_{E, M}^{(\gamma, N)} & =\sum_{q=u, d, s} Q_{q} G_{E, M}^{(q, N)} \\
G_{E, M}^{(Z, N)} & =\sum_{q=u, d, s} C_{V}^{q} G_{E, M}^{(q, N)} \\
G_{A}^{e N} & =\sum_{q=u, d, s} C_{A}^{q} G_{A}^{(q, N)}
\end{aligned}
$$

where $C_{V}^{q}$ and $C_{A}^{q}$ are the vector and axial coupling to quarks. The contribution of the three heavy flavours, has been neglected due to their larger mass. Using the charge symmetry approximation [6] under the exchange of $\mathrm{u}$ and d quarks $\left(G_{E, M}^{(u, p)}=G_{E, M}^{(d, n)}\right)$ and s quark $\left(G_{E, M}^{(s, p)}=G_{E, M}^{(s, n)}\right)$, it is possible to express the electric and magnetic quark form factors as a function of electromagnetic and weak form factors of the proton and neutron. In particular, the extraction of the strange form factor requires the measurement of electromagnetic and weak for factors through the relation :

$G_{E, M}^{s}=\left(1-4 \sin ^{2} \theta_{W}\right) G_{E, M}^{(\gamma, p)}-G_{E, M}^{(\gamma, n)}-G_{E, M}^{(Z, p)}(2)$

Experimentally, the electromagnetic nucleon form factors are obtained through a Rosenbluth separation on the elastic electron-nucleon crosssections on proton and neutron targets. .

Because the weak interaction does not conserve parity, $\mathcal{M}_{Z}$ and the weak vector nucleon form factors are accessible through the asymmetry measurement of the cross-sections associated with elastic scattering of longitudinaly polarized electrons with two opposite helicities :

$$
A_{P V}=\frac{\left|\mathcal{M}^{+}\right|^{2}-\left|\mathcal{M}^{-}\right|^{2}}{\left|\mathcal{M}^{+}\right|^{2}+\left|\mathcal{M}^{-}\right|^{2}}=A_{P V}^{0}+A_{P V}^{s}
$$

where the first term is independent of the strange form factors :

$$
\begin{array}{r}
A_{P V}^{0}=-\left(\frac{G_{F} Q^{2}}{4 \sqrt{2} \pi \alpha}\right) \frac{1}{\epsilon\left(G_{E}^{(\gamma, p)}\right)^{2}+\tau\left(G_{M}^{(\gamma, p)}\right)^{2}} \\
{\left[\epsilon G_{E}^{(\gamma, p)}\left(\left(1-4 \sin ^{2} \theta_{W}\right) G_{E}^{(\gamma, p)}-G_{E}^{(\gamma, n)}\right)\right.} \\
+\tau G_{M}^{(\gamma, p)}\left(\left(1-4 \sin ^{2} \theta_{W}\right) G_{M}^{(\gamma, p)}-G_{M}^{(\gamma, n)}\right) \\
\left.-\left(1-4 \sin ^{2} \theta_{W}\right) \epsilon^{\prime} G_{M}^{(\gamma, p)} G_{A}^{e p}\right]
\end{array}
$$

and the second one contains the contribution of $G_{E}^{s}$ et $G_{M}^{s}$ :

$$
A_{P V}^{s}=\left(\frac{G_{F} Q^{2}}{4 \sqrt{2} \pi \alpha}\right) \frac{G_{E}^{(\gamma, p)} G_{E}^{s}+\tau G_{M}^{(\gamma, p)} G_{M}^{s}}{\epsilon\left(G_{E}^{(\gamma, p)}\right)^{2}+\tau\left(G_{M}^{(\gamma, p)}\right)^{2}}
$$

with $\tau=\frac{Q^{2}}{4 M^{2}}, \epsilon=\frac{1}{1+2(1+\tau) \tan ^{2} \frac{\theta_{e}}{2}}$ and $\epsilon^{\prime}=$ $\sqrt{\tau(1+\tau)\left(1-\epsilon^{2}\right)}$. Asymmetries as small as few $10^{-6}$ can be extracted with a precision of a few $\%$ thanks to cancellation of normalization systematic errors in the ratio of the cross-section measurements.

The $G^{0}$ collaboration proposes to perform the complete separation of the electric, $G_{E}^{s}$, magnetic, $G_{M}^{s}$ and axial, $G_{A}^{e p}$, form factors for two different momentum tranfers 0.23 and $0.68(\mathrm{GeV} / \mathrm{c})^{2}$. This can be accomplished performed by varying the kinematical factors $\left(\epsilon\right.$ and $\left.\epsilon^{\prime}\right)$ at a fixed $Q^{2}$. A Forward Angle measurement has already been performed for electron scattering angles between 7 and $15^{\circ}$ for a large $Q^{2}$ range between 0.1 and $1(\mathrm{GeV} / \mathrm{c})^{2}$. This measurement is obtained by detecting the recoil protons at a fixed angle between 52 and $78^{\circ}$. Two Backward Angle measurements then have to be performed at an electron scattering angle of about $108^{\circ}$ on hydrogen and deuterium targets providing different combination of the three form factors. Two different incident beam energies of 360 and $687 \mathrm{MeV}$, corresponding to $Q^{2}$ of 0.23 and $0.63(\mathrm{GeV} / \mathrm{c})^{2}$ are used.

Even if the primary goal of PV experiments is to determine the vector form factors, they also provide a measurement of the axial form factor in electron-nucleon scattering, which can be compared to the one obtained in neutrino-nucleon scattering [7]. It can be expressed through the relation :

$$
\begin{array}{r}
G_{A}^{e N}\left(Q^{2}\right)=-\tau\left(1+R_{A}^{T=1}\right) G_{A}^{C C}\left(Q^{2}\right) \\
+\sqrt{3} R_{A}^{T=0} G_{A}^{(8)}\left(Q^{2}\right)+\left(1+R_{A}^{(0)}\right) G_{A}^{s}\left(Q^{2}\right)
\end{array}
$$

The first term, corresponding to the charged current contribution, is written as $G_{A}^{C C}\left(Q^{2}\right)=$ $g_{A} /\left(1+Q^{2} M_{A}^{2}\right)^{2}$, where $M_{A}=1.001 \pm 0.020$ and $g_{A}=1.2695 \pm 0.0029$ are obtained respectively 
from quasielastic neutrino data and neutron $\beta$ decay. Isoscalar corrections are given by $G_{A}^{(8)}$, with its value at $Q^{2}=0^{\circ} G_{A}^{(8)}(0)=\frac{1}{2 \sqrt{3}}(3 F-D)=$ $0.169 \pm 0.007$ being provided by hyperon decay. The third term corresponds to the strange contribution to the axial form factor with $G_{A}^{s}(0)=\Delta s$ measured in deep inelastic scattering and ranging from -0.14 to 0 . The three electroweak radiative correction factors $R_{A}^{T=1}=-0.24 \pm 0.24$, $R_{A}^{T=0}=-0.24 \pm 0.14$ and $R_{A}^{T=0}=-0.55$, arising from higher order diagrams, are calculated theoretically at $Q^{2}=0$ but with large uncertainty like on the isovector contribution. Except for $G_{A}^{C C}$, the $Q^{2}$ dependence of the different terms is unknown.

Three other experiments have been designed to measure the strange contribution to the vector and axial nucleon form factors. The HAPPEx experiment, which takes place in the hall A of Jefferson Laboratory, has performed Forward Angle measurements on proton and helium at $Q^{2}=0.1$, $0.48(\mathrm{GeV} / \mathrm{c})^{2}$ and has proposed a new measurement at $0.63(\mathrm{GeV} / \mathrm{c})^{2}$ [8]. The experiment $A 4$, located at the MAMI facility, has provided two Forward Angle measurements for $Q^{2}=0.1$ and $0.23(\mathrm{GeV} / \mathrm{c})^{2}$ and should take data in Backward Angle configuration in 2006 [9]. The SAMPLE experiment, which was performed at MIT Bates facility, has measured Backward Angle asymmetries on proton and deuteron for $Q^{2}=0.04$ and $0.1(\mathrm{GeV} / \mathrm{c})^{2}[10]$.

\section{3. $G^{0}$ experimental set-up in the Forward angle configuration}

Because the $G^{0}$ experiment measures very small asymmetries between 3 to $50 \mathrm{ppm}$ with a precision of few \%, it requires a very high beam intensity in conjunction with a dedicated setup of very large acceptance to achieve high statistics of about $10^{13}$ elastic events.

The polarized electrons are produced by the interaction of a circularly polarized laser on the strained GaAs photocathode, which allows for a high polarization $(\geq 70 \%)$, with the polarity reversed at $30 \mathrm{~Hz}$ frequency. The high intensity beam is then accelerated by $C E B A F$ to the nominal beam energy of $3.045 \mathrm{GeV}$. An important issue in this kind of experiment is to minimize false asymmetries induced by helicity-correlated changes in beam parameters as the charge, positions, angles and energy. It is achieved by implementing a feedback device on the beam parameters using the beam asymmetries measured in the hall.

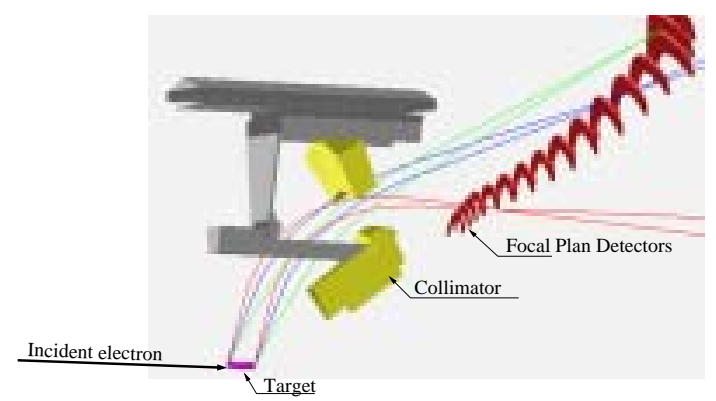

Figure 1. Trajectories of protons elastically scattered for three different $Q^{2}$.

The $G^{0}$ set-up is composed of a $20 \mathrm{~cm}$ cryogenic liquid hydrogen target located at the center of a large spectrometer, which consists of a superconducting toroidal magnet with eight sectors placed symmetrically around the beam axis. The recoil protons produced in the elastic scattering on the target, are selected in momentum within a large solid angle by the magnetic field so that their location on the focal plane depend only on the $Q^{2}$, ranging between 0.1 to $1(\mathrm{GeV} / \mathrm{c})^{2}$ (see figure 1). Collimators prevent a direct view of the target by the detectors and thus reduce the background coming from neutral particles in the detectors. The FPD detectors are composed of 16 pairs of plastic scintillators coupled to photomultipliers via light guides.

The elastic protons are discriminated from other particles (protons, pions etc ...) using timeof-flight (see Fig. 2). For this purpose specific electronics have been developed to handle the counting rates associated with each helicity state as a function of the time-of-flight on a $32 \mathrm{~ns}$ time range. The information is histogrammed during one time period $(33 \mathrm{~ms})$ for counting rates of few $\mathrm{MHz}$ with a reasonable deadtime between 5 to $20 \%$. A sampled event-by-event Fastbus acquisition of time and charge information has also been 


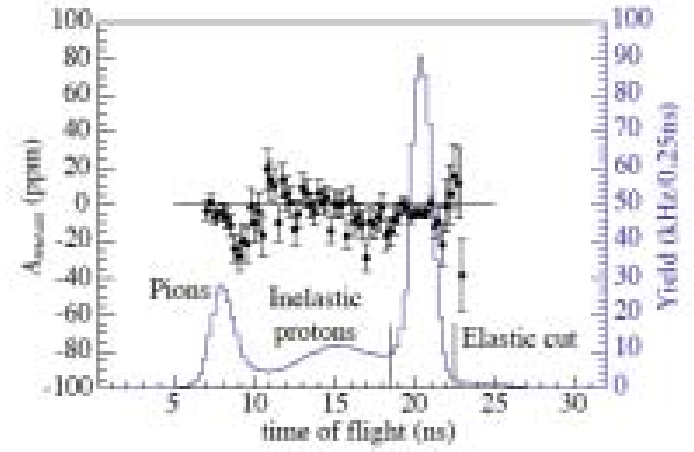

Figure 2. Measured yields and raw asymmetries as a function of time of flight for detector 8 .

used for monitoring detectors and beam parameters. More details on the experimental set-up can be found in the references $[11,12]$

\section{G0 Forward angle results}

About 700 hours of data have been recorded in the Forward angle configuration. The data analysis calculates the physics asymmetries from the measured asymmetries of the detectors counting rates normalized by the beam charge, after applying a certain number of corrections. Some corrections are related to the beam properties, including the helicity-correlated beam parameters (charge, position ...) and the beam polarization. Also the effect of leakage coming from the other beams has been corrected with good accuracy. Corrections concerning the $G^{0}$ set-up such as the effect of the electronics deadtime and the determination of the $Q^{2}$ associated to different detectors are then applied. The main correction however was due to the subtraction of the background in the asymmetries. The background, which can be seen in figure 2 , represents about 5 to $20 \%$ of the counting rates under the elastic peak in the time-offlight spectrum depending on the detector. The background correction on the asymmetries has been performed with final uncertainties ranging between 0.2 to $9 \mathrm{ppm}$. Details of the analysis including final estimates of systematic uncertainties can be found in references [13,12]. Finally, experimental asymmetries have been obtained for 18

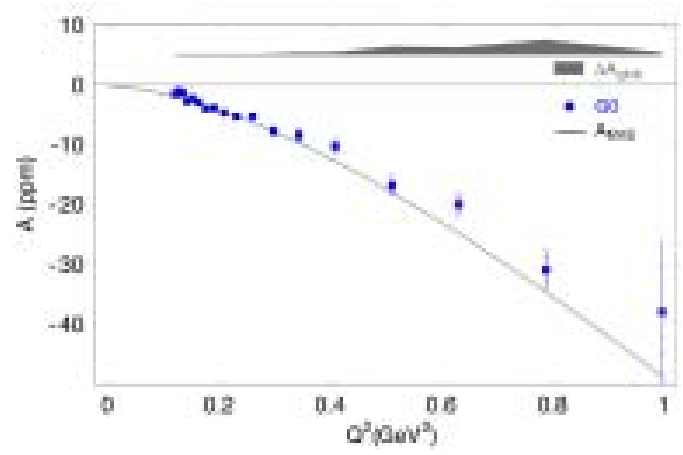

Figure 3. Physics asymmetries obtained by $G^{0}$ experiment in the Forward Angle configuration after several corrections (see reference [13]).

different values of $Q^{2}$ ranging between 0.12 to 1 $(\mathrm{GeV} / \mathrm{c})^{2}$, which are plotted in figure 3. A linear combination $G_{E}^{s}+\eta G_{M}^{s}$ is then deduced from equations 3 to 5 after subtracting the no-vectorstrange contribution from the asymmetries. As shown in figure 4 , the $G_{E}^{s}+\eta G_{M}^{s}$ data exhibit a noticeable $Q^{2}$ dependence with a strong variation close to $0.2(\mathrm{GeV} / \mathrm{c})^{2}$ and positive values at larger $Q^{2}$. It has been shown that these experimental data are not compatible with a $G_{E}^{s}+\eta G_{M}^{s}=0$ hypothesis with a $89 \%$ confidence level [13].

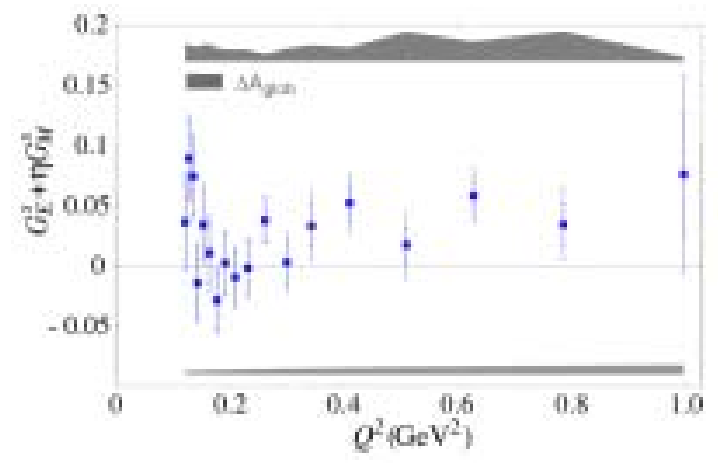

Figure 4. $G^{0}$ results on the combinaison of $G_{E}^{s}+$ $\eta G_{M}^{s}$. The gray band indicate systematic uncertainties (see reference [13]). 
The $G^{0}$ result on $G_{E}^{s}+\eta G_{M}^{s}$ can then be combined with those from other PV experiments (HAPPEX, A4, SAMPLE) to separate the electric and magnetic form factors. A fit of the data over a large range of $Q^{2}$ uses parametrization for $G_{E}^{s}=a_{2} Q^{2} /\left(1+b_{1} Q^{2}+b_{2} Q^{4}+b_{3} Q^{6}\right)$ and $G_{M}^{s}=\mu_{s} /\left(1+Q^{2} / \Lambda_{s}\right)$. The results, which are shown in figure 5 , suggest a large and positive contribution (of the order of $10 \%$ ) $G_{M}^{s}(0)$ to the magnetic moment of the nucleon and a negative contribution of $G_{E}^{s}$ (of opposite sign of the neutron electric form factor) located around 0.2 $(\mathrm{GeV} / \mathrm{c})^{2}$.

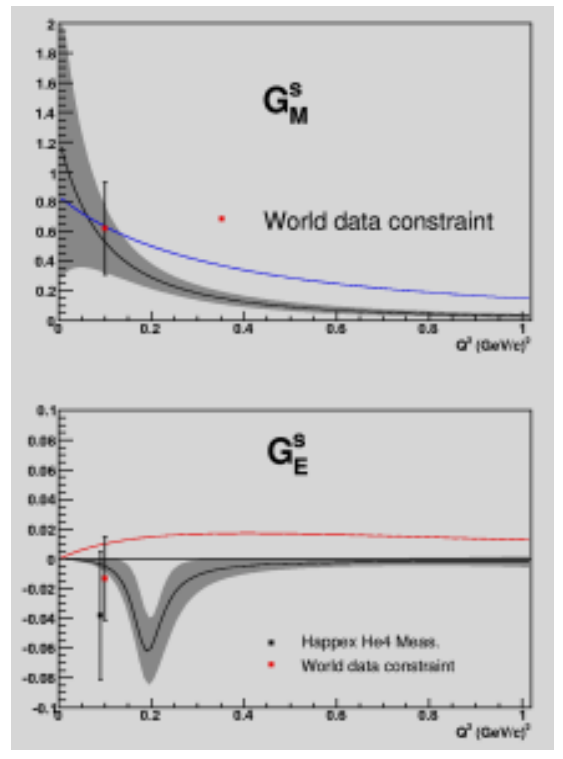

Figure 5. Parametrization of $G_{E}^{s}$ and $G_{M}^{s}$ obtained from a fit of PV experimental data (taken from reference [12]). For reference, lines indicates $10 \%$ contribution of proton magnetic form factor on the $G_{M}^{s}$ plot and $10 \%$ contribution of neutron electric form factor on the $G_{E}^{s}$ plot

Reference [14] has demonstrated that elastic $\nu p$ and $\bar{\nu} p$ cross-sections measurements and PV ep elastic asymmetries can be combined to extract $G_{E}^{s}, G_{M}^{s}$ and $G_{A}^{s}$, the neutrino data being more sensitive to the strange axial form factor. The results, using the new $G^{0}$ data combined with the old E734 data, are shown in figure 6 . The strange axial form factor $G_{A}^{s}$ is determined between 0.45 and $1(\mathrm{GeV} / \mathrm{c})^{2}$ with reasonable uncertainties but cannot be extrapolated to $Q^{2}=0$ to be compared with the result obtained in deep inelastic scattering. In the future, the FINESSE experiment [16] is expected to measure $G_{A}^{s}$ down to $Q^{2}=0.2$ $(\mathrm{GeV} / \mathrm{c})^{2}$.

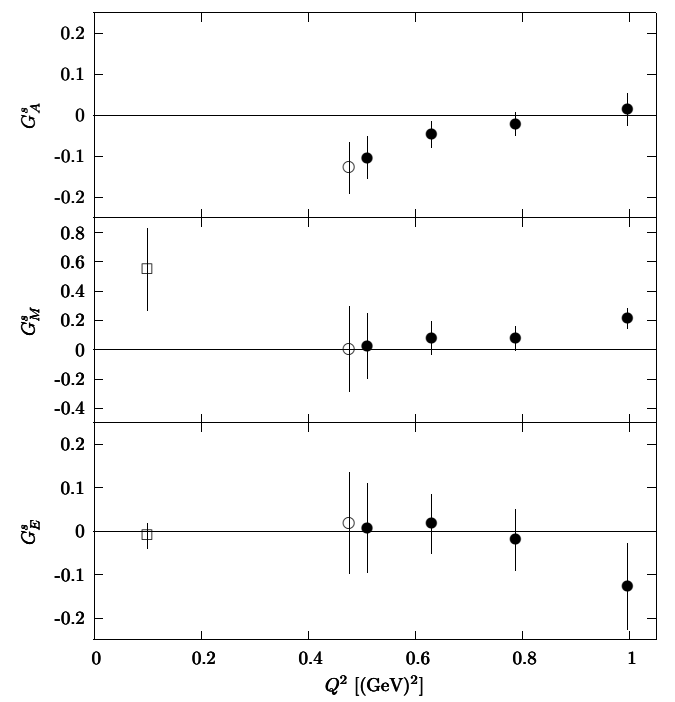

Figure 6. Results on the extraction of $G_{E}^{s}, G_{M}^{s}$ and $G_{A}^{s}$ using PV experiments including $G^{0}$ data combined with E734 neutrino data (taken from reference [15]).

\section{Summary}

The forward angle asymmetries measured in elastic electron-proton scattering obtained from the $G^{0}$ experiment have allowed the determination of a linear combination $G_{E}^{s}+\eta G_{M}^{s}$ over the largest range of momentum transfers ever achieved, between 0.12 to $1(\mathrm{GeV} / \mathrm{c})^{2}$. These 
new results, which exhibit a $Q^{2}$ dependence of $G_{E}^{s}+\eta G_{M}^{s}$, are compatible with contributions of opposite signs to the electric and magnetic form factors. When combined with the other PV experimental results, these data add new constraints on the determination of $G_{E}^{s}$ and $G_{M}^{s}$ under some hypothesis on the parametrizations of these form factors. They can can also be combined with neutrino data to extract the strange axial form factors between 0.45 and $1(\mathrm{GeV} / \mathrm{c})^{2}$.

After the turn-around of the spectrometer, a second phase of $G^{0}$ experiment will be started in 2006 to measure asymmetries in the backward angle kinematics. Data taking will be performed on hydrogen and deuterium targets for $Q^{2}=0.23$ and $0.63(\mathrm{GeV} / \mathrm{c})^{2}$ by detecting the electrons at about $108^{\circ}$. In this configuration the background, which is composed of electrons and negative pions coming from inelastic processes, will be separated using the FPD detectors in conjunction with a second hodoscope (CED). Also the $\pi^{-}$produced on hydrogen and deuterium targets will be separated from electrons with the help of a Čerenkov counter. The $C E D-F P D$ coincidences, enabled by the Čerenkov signal, will then provide asymmetries not only for the elastic scattering but also for the $N-\Delta$ inelastic transition process [17].

\section{Acknowledgments}

I would like to thank the organisers of NUINT05 for their hospitality. I would like also to thank all $G^{0}$ collaborators, wishing successful data taking for 2006. The $G^{0}$ experiment is supported by DOE (U.S.), CNRS/IN2P3 (France), NSERC (Canada) and NSF (U.S.).

\section{REFERENCES}

1. D. Kaplan and A. Manohar, Nucl. Phys. B310 (1988) 527.

2. R.D. McKeown, Phys. Lett. B219 (1989) 140.

3. D.H. Beck, Phys. Rev. D39 (1989) 3248.

4. D.H. Beck and R.D. McKeown, Ann. Rev. Part. Sci. 51 (2001) 189.

5. K.S. Kumar and P.A. Souder, Prog. in Part. and Nucl. Phys. 45 (2000) S33.
6. G.A. Miller, Phys. Rev. C57 (1998) 1492.

7. E.J. Beise, in PAVI04 proceeding, Eur. Phys. J A24S2 (2005) 43.

8. K.A. Aniol et al., Phys. Rev. C69 (2004) 065501; K.A. Aniol et al., nucl-ex/0506010; K.A. Aniol et al., nucl-ex/0506011.

9. F.E. Maas et al., Phys. Rev. Lett. 93 (2004) 022002; F.E. Maas et al., Phys. Rev. Lett. 94 (2005) 152001.

10. D. Spayde et al., Phys. Lett. B583 (2004) 79; T.M. Ito et al., Phys. Rev. Lett. 92 (2004) 102003.

11. P. Roos for the $G^{0}$ collaboration, Eur. Phys. J A24S2 (2005) 59.

12. B. Guillon, PHD thesis, UJF Grenoble, LPSC 05-89 (2005).

13. D.S. Armstrong et al., Phys. Rev. Lett. 95 (2005) 092001.

14. S.F. Pate, Phys. Rev. Lett. 92 (2004) 082002; S.F. Pate, Eur. Phys. J A24S2 (2005) 67.

15. S.F. Pate et al., hep-ex/0512032.

16. FINeSSE proposal, B.T. Fleming, R. Tayloe et al., hep-ex/0402007.

17. S. Wells et al., Jefferson Lab Proposal E04101. 